\title{
Nonlinear Stability and Boundedness of Approximately Symmetric Large-Scale Systems *
}

\author{
Bill Goodwine* \\ * Department of Aerospace 83 Mechanical Engineering \\ University of Notre Dame, Notre Dame, Indiana USA \\ e-mail: bill@controls.ame.nd.edu.
}

\begin{abstract}
:
Stability and convergence properties of large-scale integrated systems are essential aspects of the development of truly useful and well-designed cyber-physical systems (CPS). Because of the desire for flexible, adaptive and reactive cyber-physical systems, i.e., global operation, nonlinear analyses and tools are especially important in CPS. Hence, Lyapunov methods are at the core of many critical control methodologies for such systems. This paper considers Lyapunov stability for approximately symmetric systems. Many robotic systems, such as swarms and fleets of mobile robots, distributed sensor networks, highly integrated cyber-physical systems, etc., are comprised many identical interacting agents, and our prior work has developed computationally efficient stability analyses of the control and dynamics of such symmetric systems. This paper extends those results to the important case where all the agents are not identical, which is important for real-world applications where it is not possible to have exactly identical agents. Importantly, these results do not require the components to have small differences. However, the bounds on the nature of the solutions will obviously depend on how different the agents are.
\end{abstract}

Keywords: Symmetric systems, Lyapunov stability, nonlinear systems, robotics, distributed control

\section{INTRODUCTION}

The use of multi-agent systems has a transformative potential for the ubiquitous use of automated systems in real-world applications. This partially is due to: 1) robustness, because if a small subset of agents fail, the overall performance of a large-scale multi-agent system is not likely to be significantly reduced, 2) adaptability and agility, because, in addition to any adaptability built into an individual agent's control, significant adaptability and agility in behavior may emerge from the coordinated efforts of the agents, significantly beyond what is possible for a single agent, and 3) economics, because in many applications it may be simultaneously cheaper and more effective to accomplish a task using multiple components than a single more sophisticated one.

Despite these advantages, other than sensor networks, actually deployed cooperating agent systems are not yet ubiquitous. This is partly because their promise depends on a critical control aspect, which is that in order to bring about the desired system behavior, coordination among the agents is necessary, which is a difficult theoretical and technological control problem. One aspect of this difficulty is that if the agents are coordinating their behavior the system has a very high dimensional and coupled state space, making analysis and design of the system difficult.

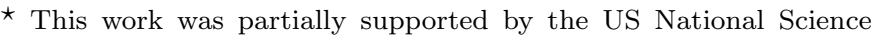
Foundation under the CPS Large Grant No. CNS-1035655.
Many recent research efforts have attempted to address this problem. Some efforts have focused on compositionality and composability wherein the relationship between system properties and the properties of individual components is determined (Sztipanovits et al. (2012); Julliand et al. (2007)). While the results in this paper are general and apply to a broad class of problems, the most natural focus in robotics would be on problems such as formation control and consensus. Formation control has a natural relationship with Lyapunov methods in that potential functions are an appealing means to define a formation. Of course there is a vast literature on formation control, and a representative set of references include: Jadbabaie et al. (2003); Ren et al. (2007); Fax and Murray (2004); Rimon and Koditschek (1992); Leonard and Fiorelli (2001); Olfati-Saber and Murray (2002) and the book by Kumar et al. (2005).

Similarly, in the controls community, consensus has received much attention and is an important subject because of the need for distributed agents to reach a consensus on many things ranging from collected sensor data to motions for flocking behavior. While it is emphasized that our results are much more broadly applicable than just to the consensus problem, the main example in this paper is a nonlinear consensus one. In contrast to most work in consensus which focuses on the interconnectedness of the system (see for example, Olfati-Saber and Murray (2003); Ren et al. (2005)), we focus on system properties which are invariant with a pre-defined regularity in the system 


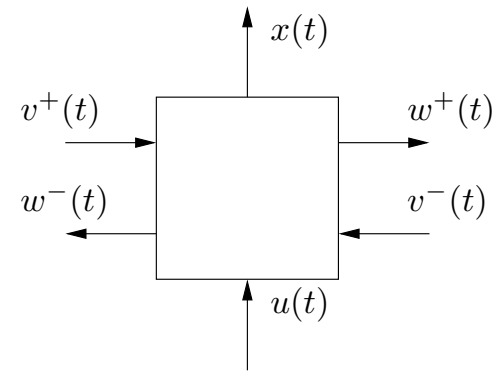

Fig. 1. System building block in one spatial dimension.

structure with an emphasis on robustness when the system is not exactly symmetric.

Prior work of the author has focused on control of symmetric systems: Goodwine and Antsaklis (2013); McMickell and Goodwine (2003b, 2002, 2003a, 2007, 2001); McMickell et al. (2003). The precise meaning of a symmetric system will be defined subsequently, but the simplest example is a system that is composed of identical agents interacting with each other in a highly-structured manner. In such a case, it makes sense that the equations of motion for the system will exhibit significant structure which may be exploited for analysis and design in control.

One shortcoming of the existing work is that it requires the system to be exactly symmetric in that all the agents must either be identical (at least diffeomorphically). This work is part of a series of efforts to extend those results to the case where the agents in the system are only approximately symmetric. This will encompass the realities that it is not possible in the real world to have agents that are exactly identical as well as the fact that it may be desirable for the agents to differ. Closely related current work by the author includes Goodwine (2013).

\section{SYMMETRIC AND APPROXIMATELY SYMMETRIC SYSTEMS}

This section is a summary of our results from Goodwine and Antsaklis (2013) and gives an overview of symmetric systems and the relationship among symmetric systems with different numbers of components. It also extends these to allow for additive symmetry-breaking terms to each component.

Consider the "building block" for symmetric systems illustrated in Figure 1. The $w^{+}(t)$ and $w^{-}(t)$ signals are the outputs from the component and $u, v^{-}(t)$ and $v^{+}(t)$ are the inputs. The signals $v^{ \pm}$represent coupling with the other components and $u$ are the control inputs. We wish to consider fully nonlinear symmetric systems of the form

$$
\begin{aligned}
\dot{x}_{i}(t) & =f_{i}\left(x_{i}(t)\right)+\sum_{j=1}^{m_{i}} g_{i, j}\left(x_{i}(t)\right) u_{i, j}(t) \\
w_{i}^{-}(t) & =w_{i}^{-}\left(x_{i}(t)\right), \quad w_{i}^{+}(t)=w_{i}^{+}\left(x_{i}(t)\right) .
\end{aligned}
$$

If the system is controlled via feedback, the control input for component $i$ in Equation 1 may depend on the outputs from the neighbors, which can be expressed by

$$
u_{i, j}(t)=u_{i, j}\left(x_{i}(t), w_{i-1}^{+}\left(x_{i-1}(t)\right), w_{i+1}^{-}\left(x_{i+1}(t)\right)\right) .
$$

The block in Figure 1 has only + and - inputs and outputs, so we consider systems defined on groups in

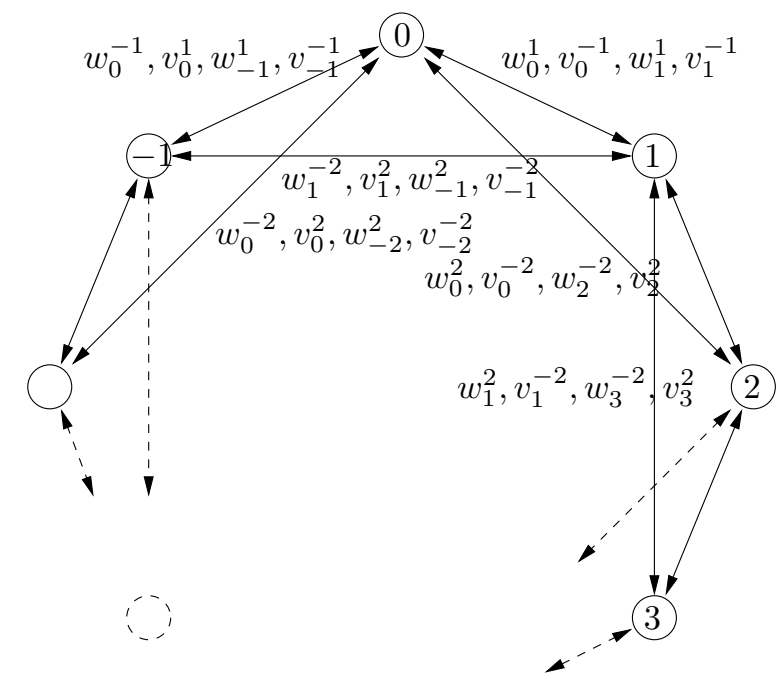

Fig. 2. System topology for Example 1.

order to allow systems with a more general interconnection structure. Recall, that a group is a set, $G$ with

(1) a binary associative operation, $G \times G \rightarrow G$,

(2) an identity element $e$ such that $e g=g e=g$ for all $g \in G$, and

(3) for every $g \in G$ there exists an element $g^{-1} \in G$ such that $g g^{-1}=g^{-1} g=e$.

Let $|G|$ denote the number of elements in a set $G$.

Following the development in Recht and D'Andrea (2004), interconnections in systems we consider will be represented by a set of generators, denoted by $X$. If $X$ is a subset of a group $G$, then the smallest subgroup of $G$ containing $X$ is called the subgroup generated by $X$. For the rest of this paper assume that $G$ itself is the group generated by $X$ and that if $s \in X$, then $s^{-1} \in X$ as well. Relations define constraints among the generators, and are of the form $s_{1} s_{2} \ldots s_{m}=e$ for $s_{1}, \ldots, s_{m} \in X$. Finally, we represent systems by a Cayley graph, which is a graph with vertices that are the elements of a group, $G$, generated by the subset $X$, with a directed edge from $g_{1}$ to $g_{2}$ if $g_{2}=s g_{1}$ for some $s \in X$ (see Rotman (1995)). A directed edge from node $g_{1}$ to $g_{2}$ represents that a coupling input to $g_{2}$ is equal to an output from $g_{1}$.

The following example will be developed throughout this paper. It has a similar interconnection structure to the formation control problem we have published in Goodwine and Antsaklis (2013), but the dynamics are for a consensus problem rather than formation control.

Example 1. Consider the system with the Cayley graph illustrated in Figure 2. Each vertex has edges connecting to four other vertices and hence the system is generated by four elements. Let $g$ denote a vertex, i.e., $g \in$ $\{-2,-1,0,1, \ldots, N-3\}=G$. Consider the generators $X=\{-2,-1,1,2\}$, the group operation to be addition and the relation $s^{N}=e=0$. This relation makes the group operation of addition to be $\bmod N$, and hence the group is the quotient of the set of integers $\mathbb{Z}$ where elements of $\mathbb{Z}$ that differ by an integer multiple of $N$ are equivalent.

Next, we develop some notation. For a system on the group $G$ with generators $X=\left\{s_{1}, s_{2}, \ldots, s_{|X|}\right\}$, let $x_{g}$ denote the 
states corresponding to $g \in G, X g=\left\{s_{1} g, s_{2} g, \ldots, s_{|X|} g\right\}$ denote the neighbors of component $g \in G, x_{X g}$ denote the states of the neighbors of $g \in G$, and $x_{X X g}$ denote the states of the neighbors of the neighbors, etc. For a component $g$, let $\left\{w_{g}^{s_{1}}, w_{g}^{s_{2}}, \ldots, w_{g}^{s_{|X|}}\right\}$ denote of outputs, and correspondingly let $\left\{v_{g}^{s_{1}}, v_{g}^{s_{2}}, \ldots, v_{g}^{s_{|X|}}\right\}$ denote the inputs. In this more general setting, the dynamics of a component, $g \in G$ are represented by

$$
\begin{aligned}
& \dot{x}_{g}(t)= f_{g}\left(x_{g}(t)\right) \\
&+\sum_{j=1}^{m_{g}} g_{g, j}\left(x_{g}(t)\right) u_{g, j}\left(x_{g}(t), v_{g}^{s_{1}}(t), \ldots, v_{g}^{s_{|X|}}(t)\right) \\
& w_{g}^{s}(t)= w_{g}^{s}\left(x_{g}(t)\right), \\
& \text { for all } s \in X .
\end{aligned}
$$

Definition 1. A system with with dynamics given by Equation 3 has periodic interconnections if

$$
v_{g}^{s}(t)=w_{s^{-1} g}^{s}\left(x_{s^{-1} g}(t)\right),
$$

for all $g \in G$ and $s \in X$. Furthermore, if

$$
\begin{aligned}
f_{g_{1}}(x) & =f_{g_{2}}(x), & g_{g_{1}, j}(x) & =g_{g_{2}, j}(x), \\
w_{g_{1}}^{s}(x) & =w_{g_{2}}^{s}(x), & m_{g_{1}} & =m_{g_{2}}=m
\end{aligned}
$$

for all $s \in X, g_{1}, g_{2} \in G, x \in \mathbb{R}^{n}$ and $j \in\{1, \ldots, m\}$, then $G$ has symmetric components. Finally, if the control laws also satisfy

$$
\begin{aligned}
& u_{g_{1}, j}\left(x_{1}, w_{s_{1}^{-1} g_{1}}^{s_{1}}\left(x_{2}\right), \ldots, w_{s_{|X|}^{-1} g_{1}}^{s_{|X|}}\left(x_{|X|+1}\right)\right)= \\
& u_{g_{2}, j}\left(x_{1}, w_{s_{1}^{-1} g_{2}}^{s_{1}}\left(x_{2}\right), \ldots, w_{s_{|X|}^{-1} g_{2}}^{s_{|X|}}\left(x_{|X|+1}\right)\right)
\end{aligned}
$$

for all $g_{1}, g_{2} \in G, j \in\{1, \ldots, m\}, s \in X$ and $\left(x_{1}, x_{2}, \ldots, x_{|X|+1}\right) \in \mathbb{R}^{n} \times \mathbb{R}^{n} \times \cdots \times \mathbb{R}^{n}$ then the system is called a symmetric system on $G$.

Remark 1. Definition 1 provides the natural mathematical requirements for the agents to be "the same". Equation 4 simply requires that the coupling inputs to agent $g$ are the outputs from the corresponding neighbors, the equations in 5 require the dynamics of each agent to be identical and Equation 6 requires the feedback control laws be identical for each agent.

Example 2. We will return to Example 1 and add dynamics to each component. We will take the equation of motion for the $i$ th agent to be in a modified nonlinear consensus form

$$
\dot{x}_{i}=u_{i}=k \sum_{j \in \mathcal{N}}\left(x_{j}-x_{i}\right)^{3}-x_{i}
$$

where $\mathcal{N}=\{i-2, i-1, i+1, i+2\}(\bmod N)$. We will show this is a symmetric system.

In Example 1 we showed that this be represented by the graph illustrated in Figure 2 with

$$
G=\{-2,-1,0,1,2, \ldots, N-3\},
$$

the group operation to be addition and $X=\{-2,-1,1,2\}$ with the relation $s^{N}=0, N \geq 5$. Also observe from Equation 7, the control for robot $i$ depends on its own state as well as the states for robots $i-2, i-1, i+1$ and $i+2$, which are equivalent to the four generators. Hence, define all four outputs for robot $i$ to be the vector of the robot's position, i.e., $w_{i}^{s}=x_{i}$ where $s \in X=\{-2,-1,1,2$,$\} . De-$ fine the inputs to $i \in\{-2,-1, \ldots, N-3\}$ to be $v_{i}^{s}=x_{i+s}$, $s \in\{-2,-1,1,2\}$ which satisfies Equation 4 . The dynamics, as given in Equation 7 satisfy Equation 5. Finally, the feedback law given in Equation 7 satisfies Equation 6 . Because these hold for all $i \in\{-2,-1,0, \ldots, N-3\}$ the system is a symmetric system.

Now, we will define two systems to be equivalent if they are symmetric with identical components which are interconnected in the same manner, but possibly with a different number of components. This will allow us to either "build up" or reduce the size of a system by adding or removing components, respectively. Because interconnections are defined by the generators if the two systems have the same generators, then any new agent will have the same relationship with its neighbors.

Definition 2. Two symmetric systems on the finite groups $G_{1}$ and $G_{2}$ are equivalent if $G_{1}$ and $G_{2}$ are generated by the same set of generators, $X$,

$$
\begin{aligned}
f_{g_{1}}(x) & =f_{g_{2}}(x), \quad g_{g_{1}, j}(x)=g_{g_{2}, j}(x), \\
w_{s^{-1} g_{1}}^{s}(x) & =w_{s^{-1} g_{2}}^{s}(x)
\end{aligned}
$$

and the feedback part of the control laws satisfy

$$
\begin{aligned}
& u_{g_{1}, j}\left(x_{1}(t), w_{s_{1}^{-1} g_{1}}^{s_{1}}\left(x_{2}(t)\right), \ldots, w_{s_{|X|}^{-1} g_{1}}^{s_{|X|}}\left(x_{|X|+1}(t)\right)\right)= \\
& u_{g_{2}, j}\left(x_{1}(t), w_{s_{1}^{-1} g_{2}}^{s_{1}}\left(x_{2}(t)\right), \ldots, w_{s_{|X|}^{-1} g_{2}}^{s_{|X|}}\left(x_{|X|+1}(t)\right)\right)
\end{aligned}
$$

for all $g_{1} \in G_{1}, g_{2} \in G_{2}, s \in X, x \in \mathbb{R}^{n}$, $\left(x_{1}, x_{2}, \ldots, x_{|X|+1}\right) \in \mathbb{R}^{n} \times \mathbb{R}^{n} \times \cdots \times \mathbb{R}^{n}$ and $j \in$ $\{1, \ldots, m\}$ where $m=m_{g_{1}}=m_{g_{2}}$.

Example 3. Continuing Example 2 consider two systems with components that satisfy Equation 7 and components belonging to

$$
\begin{aligned}
& G_{1}=\{-2,-1,0,1,2, \ldots, N-3\} \\
& G_{2}=\{-2,-1,0,1,2, \ldots, M-3\}
\end{aligned}
$$

where $M>N$. Because the dynamics of all the components are identical and the feedback definitions are identical, these systems are equivalent. Both have generating sets $X=\{-2,-1,1,2\}$ with the only difference being the relation for $G_{1}$ is $s^{N}=0$ and the relation for $G_{2}$ is $s^{M}=0$.

Finally, we define an approximately symmetric system.

Definition 3. Consider

$$
\begin{aligned}
\dot{x}_{g}(t)= & f_{g}\left(x_{g}(t)\right)+p_{g}\left(x_{G}(t)\right) \\
& +\sum_{j=1}^{m_{g}} g_{g, j}\left(x_{g}(t)\right) u_{g, j}\left(x_{g}(t), v_{g}^{s_{1}}(t), \ldots, v_{g}^{s_{|X|}}(t)\right) \\
w_{g}^{s}(t)= & w_{g}^{s}\left(x_{g}(t)\right),
\end{aligned}
$$

for all $s \in X$. If, in the absence of the $p_{g}$ vector field, the system satisfies all the requirements of a symmetric system, then it is an approximately symmetric system. The system obtained by setting $p_{g}=0$ for all $g \in G$ is called the corresponding symmetric system. If two different approximately symmetric have equivalent corresponding symmetric systems, then they are called equivalent approximately symmetric systems.

Note the form of $p_{g}$ is very general and hence can encompass additive perturbations on the drift term, $f_{g}$, on the control vector fields $g_{g}$ or on the control inputs.

Example 4. Returning to Example 3, adding a perturbation to each agent's dynamics of the form 


$$
\dot{x}_{i}=k \sum_{j \in \mathcal{N}}\left(x_{j}-x_{i}\right)^{3}-x_{i}+p_{i}\left(x_{-2}, \ldots, x_{N-3}\right) .
$$

This is an approximately symmetric system regardless of the specific form of $p_{i}$.

\section{STABILITY AND BOUNDEDNESS OF APPROXIMATELY SYMMETRIC SYSTEMS}

The main result in this paper is based on incorporating the following result into the symmetric context. There are many results on boundedness of solutions for systems with perturbed dynamics and this paper presents one of the possible ways to address the problem.

Theorem 2. Consider

$$
\dot{x}=f(x)
$$

Suppose that there exists a Lyapunov function, $V(x)$ defined on $0 \leq t<\infty,\|x\| \geq R$ where $R$ may be large, which satisfies the following conditions:

(1) $\alpha(\|x\|) \leq V(x) \leq \beta(\|x\|)$ where $\alpha(r)$ is continuously increasing and radially unbounded and $\beta(r)$ is continuously increasing, and

(2) the derivative, $\dot{V}$ satisfies

$$
\frac{\partial V}{\partial x}(x) f(x) \leq 0
$$

Then the solutions of Equation 12 are uniform-bounded.

Proof. This is a corollary from Yoshizawa (1966) to Theorem 10.12 but simplified with autonomous dynamics. Results along a similar line are also in Khalil (2002).

The idea, of course, is that if $\dot{V}<0$ for large $\|x\|$ but not necessarily so for $\|x\| \leq R$, then any solution starting with large magnitude initial conditions will decrease at least to near $\|x\|=R$.

The idea of the main result in this paper is that if an exactly symmetric system has very stable dynamics, then the bounded perturbed terms result in, at most, solutions which deviate from asymptotically stable behavior by an amount determined by the perturbation terms. Furthermore, this result holds for the entire equivalence class of systems, so a robotics control engineer only needs to check one system in the entire class to ensure stable operation of the entire class of systems.

Proposition 3. Given a symmetric system on a finite group $G$ with generators $X$, let $\mathcal{D}_{G} \subset \mathbb{R}^{n} \times \cdots \times \mathbb{R}^{n}(|G|$ times $)$ be an open domain,

$$
\delta=\sup _{g \in G, x \in \mathcal{D}_{G}}\left\|p_{g}\left(x_{G}\right)\right\|
$$

and assume there is a smooth function $V_{G}: \mathcal{D}_{G} \rightarrow \mathbb{R}$, $\alpha_{1}\left\|x_{G}\right\|^{2} \leq V_{G}\left(x_{G}\right) \leq \alpha_{2}\left\|x_{G}\right\|^{2}$ for all $x_{G} \in \mathcal{D}_{G}$ and $\alpha_{1}, \alpha_{2}>0$ such that:

(1) $V_{G}$ may be expressed as the sum of terms corresponding to each component where

$$
V_{g}: \underbrace{\mathbb{R}^{n} \times \cdots \times \mathbb{R}^{n}}_{1+|X| \text { times }} \rightarrow \mathbb{R}
$$

$$
\begin{aligned}
& V_{G}\left(x_{G}\right)=\sum_{g \in G} V_{g}\left(x_{g}, x_{X g}\right) \\
& =\sum_{g \in G} V_{g}\left(x_{g}, w_{s_{1}^{-1} g}^{s_{1}}\left(x_{s_{1}^{-1} g}\right), \ldots, w_{s_{|X|}^{-1} g}^{s_{|X|}}\left(x_{s_{|X|}^{-1} g}\right)\right),
\end{aligned}
$$

for all $x \in \mathcal{D}_{G}$,

(2) the individual functions corresponding to each component in $G$ are equal as functions, i.e.,

$$
V_{g_{1}}=V_{g_{2}}=V
$$

for all $g_{1}, g_{2} \in G$, and

(3) for any one of the $g \in G$,

$$
\left\|\frac{\partial V_{G}}{\partial x_{g}}\left(x_{G}\right)\right\| \leq \alpha_{3}\left\|x_{g}\right\|
$$

and

$$
\begin{array}{r}
\frac{\partial V_{G}}{\partial x_{g}}\left(x_{G}\right)\left(f_{g}\left(x_{g}\right)+\sum_{j=1}^{m} g_{g, j}\left(x_{g}\right) u_{g, j}\left(x_{g}, x_{X g}\right)\right) \\
\leq-\alpha_{4}\left\|x_{g}\right\|^{2}
\end{array}
$$

for some $\alpha_{3}, \alpha_{4}>0$.

Then the solution to any system in the equivalence class is uniform-bounded.

Proof. First we show that the conditions for Theorem 2 are satisfied and then we will show that any equivalent system on $\hat{G}$ is such that Theorem 2 is also satisfied.

Because the Lyapunov functions corresponding to each component are identical, we may take

$$
\mathcal{D}_{G}=\underbrace{\mathcal{D} \times \cdots \times \mathcal{D}}_{|G| \text { times }}
$$

for some subset $\mathcal{D} \subset \mathbb{R}^{n}$. Note that for $h \in G$, because only $V_{h}$ and its neighbors depend on $x_{h}$,

$$
\begin{aligned}
\frac{\partial V_{G}}{\partial x_{h}}\left(x_{G}\right) & =\frac{\partial}{\partial x_{h}}\left(\sum_{g \in G} V_{g}\left(x_{g}, x_{X g}\right)\right) \\
& =\frac{\partial}{\partial x_{h}}\left(\sum_{s=e, s \in X} V_{s h}\left(x_{s h}, x_{X s h}\right)\right)
\end{aligned}
$$

where $e$ is the identity element in $G$. Hence,

$$
\begin{gathered}
\dot{V}_{G}\left(x_{G}\right)=\sum_{g \in G}\left[\frac{\partial}{\partial x_{g}}\left(\sum_{s=e, s \in X} V_{s g}\left(x_{s g}, x_{X s g}\right)\right)\right. \\
\left.\left(f_{g}\left(x_{g}\right)+\sum_{j=1}^{m} g_{g, j}\left(x_{g}\right) u_{g, j}\left(x_{g}, x_{X g}\right)+p_{g}\left(x_{G}\right)\right)\right] .
\end{gathered}
$$

We will show that without $p_{g}$ every term in the series in Equation 17 is equal to every other term as functions. Hence, because the domains of each function are restricted to the same range of values, then satisfaction of Equation 16 by one of them implies the same for all of them.

Consider any two $g_{1}, g_{2} \in G$. Because of the definition of a symmetric system, $f_{g_{1}}=f_{g_{2}}$ and $g_{g_{1}, j}=g_{g_{2}, j}$ as vector fields (Equation 8) and $u_{g_{1}, j}=u_{g_{2}, j}$ as functions (Equation 9). Finally, if we define the mappings corresponding to the differentials by 


$$
\begin{gathered}
D_{g} V: \mathcal{D} \times \cdots \times \mathcal{D} \rightarrow \mathbb{R}^{n} \\
D_{g} V\left(x_{g}, x_{X g}, x_{X X g}\right)=\frac{\partial}{\partial x_{g}}\left(\sum_{s=e, s \in X} V_{s g}\left(x_{s g}, x_{X s g}\right)\right),
\end{gathered}
$$

the differentials corresponding to different components are equal as differentials i.e., $D_{g_{1}} V=D_{g_{2}} V$. Hence, as functions, each term in the square brackets (without the $p_{g}$ vector fields) are equal, and because the domain of each is restricted to the same set of values, each term satisfies Equation 16.

Thus, inequalities 15 and 16 give

$$
\begin{aligned}
\dot{V}_{G}\left(x_{G}\right) & \leq \sum_{g \in G}\left(-\alpha_{4}\left\|x_{g}\right\|^{2}-\frac{\partial V_{G}}{\partial x_{g}} p_{g}\left(x_{G}\right)\right) \\
& \leq \sum_{g \in G}\left(-\alpha_{4}\left\|x_{g}\right\|^{2}+\alpha_{3} \delta\left\|x_{g}\right\|\right) .
\end{aligned}
$$

Because $\mathcal{D}_{G}$ is the Cartesian product of the subspaces corresponding to each $g \in G,\left\|x_{G}\right\|^{2}=\sum\left\|x_{g}\right\|^{2}$. Thus, there must be at least one component such that, $\left\|x_{g}\right\|>$ $\left\|x_{G}\right\| / \sqrt{|G|}$. Because each term is a quadratic, there will be a magnitude $\left\|x_{g}\right\|$ that maximizes its corresponding term in the sum. Hence, there exists a magnitude, $R$, for which if $\left\|x_{G}\right\|>R$ even if all the remaining terms are such that $\left\|x_{g}\right\|$ maximize the corresponding term in the sum, the remaining term is sufficiently large so that the overall sum is negative. Hence, for $\left\|x_{G}\right\|>R$, the terms of Theorem 2 are satisfied.

For any equivalent system, hence with the same generators, all the arguments based on symmetry still apply. The only difference may be a different bound on $\left\|x_{G}\right\|$ due to the different number of components.

Remark 4. The conditions expressed in Inequalities 15 and 16 are stricter than they might initially appear. The lefthand sides of each are functions of $\left(x_{g}, x_{X g}\right)$ while the right-hand sides are a function only of $x_{g}$. Hence, any contribution from the states of the neighbors must either cancel or be strictly negative.

In words, what Proposition 3 provides is that if the corresponding symmetric system for an approximately symmetric system has the right stability properties, then

- the perturbed system has bounded solutions with a known bound, and,

- any equivalent approximately symmetric system also has bounded solutions if the perturbation terms are bounded.

\section{EXAMPLE}

We will illustrate the application of the results to the approximately symmetric system from Example 4. We will consider two equivalent symmetric systems one with five agents and one with fifteen agents. In each case, the equations of motion are given by Equation 11 with $k=1$ and we take the perturbation terms to be of the form

$$
p_{i}(x)=k_{i} \tan ^{-1}\left(x_{2}\right)
$$

with $k_{1}=3, k_{2}=6$ and $k_{4}=-9$ and the rest of the $k_{i}=0$. With these perturbation terms, $\delta=\sqrt{126} \pi / 2 \approx 17.6$.

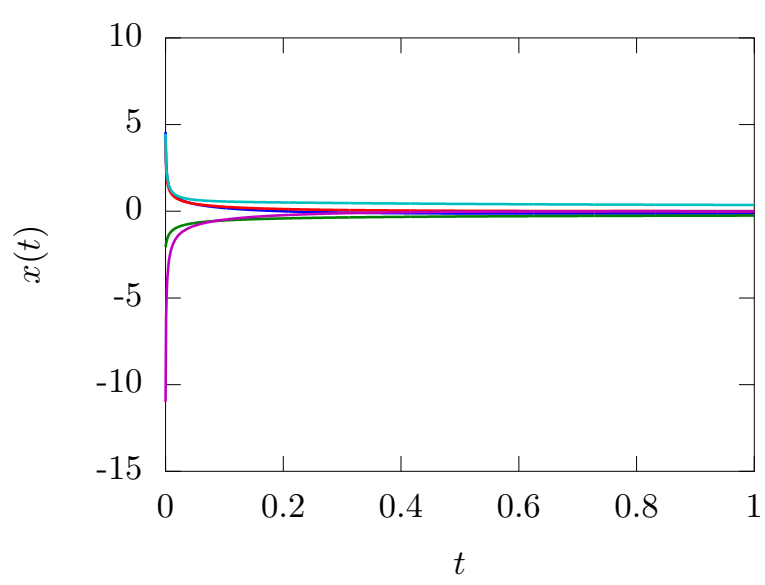

Fig. 3. Bounded solutions for approximately symmetric five-agent system.

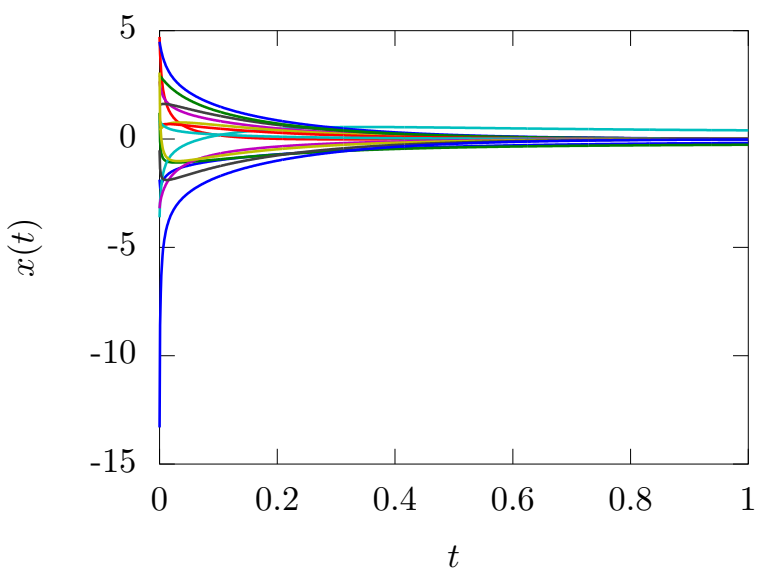

Fig. 4. Nonsymmetric consensus bounded behavior for 15agent system.

Let

$$
V=\frac{1}{2}\|x\|^{2}=\frac{1}{2} \sum_{i=-2}^{N-3} x_{i}^{2}
$$

which is of the form of Equation 13. Then for the $i$ th agent

$$
\begin{aligned}
\dot{V}_{i} & =x_{i} \dot{x}_{i}=x_{i}\left(\sum_{j \in \mathcal{N}}\left(x_{j}-x_{i}\right)^{3}-x_{i}+p_{i}\right) \\
& =\frac{1}{2} \sum_{j \in \mathcal{N}}\left(x_{i}\left(x_{j}-x_{i}\right)^{3}+x_{j}\left(x_{i}-x_{j}\right)^{3}\right)-x_{i}^{2}+x_{i} p_{i} \\
& =-\frac{1}{2} \sum_{j \in \mathcal{N}}\left(x_{j}-x_{i}\right)^{4}-x_{i}^{2}+x_{i} p_{i} .
\end{aligned}
$$

Note that if the perturbation term is removed, Equation 16 is satisfied. Also, $\frac{\partial V}{\partial x_{i}}=x_{i}$, so the bound is satisfied with $\alpha_{3}=1$. Hence, all the conditions for ultimate-bounded are satisfied.

Solutions for a five-agent system are illustrated illustrated in Figure 3. By Proposition 3, these results must hold for any equivalent system as well. For a fifteen agent system with the same perturbation terms on agents one, two and four, the solution is illustrated in Figure 4. 


\section{CONCLUSIONS AND FUTURE WORK}

This paper presented results which provide a guaranteed bound on solutions for approximately symmetric systems. It is an important extension of existing work in the literature on symmetric systems in that it allows for a much broader application of the results because it does not require an exactly symmetric system. As long as the system possesses an underlying symmetric structure which has asymptotically stable associated dynamics, then perturbations (not necessarily small) may prevent asymptotic stability, but are guaranteed to not produce solutions which grow unbounded. Importantly, because of the underlying symmetric structure, these results hold for an entire equivalence class of approximately symmetric systems.

Future work is focused on several related issues. First, more structural requirements on the perturbation will allow for stronger results, such as stability and asymptotic stability, as opposed to boundedness, in the presence of symmetry-breaking perturbations. Second, the work in this paper focused on systems with a stable equilibrium. Set-based results, based on LaSalle's theorem or related invariance principles, are an important focus for investigation because many robotic formation control problems do not have a single, stable equilibrium, but rather a set of an infinite number acceptable formations.

\section{REFERENCES}

Fax, J.A. and Murray, R.M. (2004). Information flow and cooperative control of vehicle formations. IEEE Transactions on Automatic Control, 49(9), 1465-1476.

Goodwine, B. (2013). Compositional stability of approximately symmetric systems: Initial results. In Proceedings of the 2013 Mediterranean Conference on Control $\&$ Automation (MED), 1470-1476. IEEE.

Goodwine, B. and Antsaklis, P. (2013). Multi-agent compositional stability exploiting system symmetries. Automatica, 49(11), 3158 - 3166.

Jadbabaie, A., Lin, J., and Morse, A.S. (2003). Coordination of groups of mobile autonomous agents using nearest neighbor rules. IEEE Transactions on Automatic Control, 48(6), 988-1001.

Julliand, J., Mountassir, H., and Oudot, E. (2007). Composability, compatibility, compositionality: automatic preservation of timed properties during incremental development. Technical report, UFR Sciences et Techniques.

Khalil, H.K. (2002). Nonlinear Systems. Prentice Hall, third edition.

Kumar, V., Leonard, N., and Morse, A.S. (eds.) (2005). Cooperative Control, volume 309 of Lecture Notes in Control and Information Sciences. Springer.

Leonard, N.E. and Fiorelli, E. (2001). Virtual leaders, artificial potentials and coordinated control of groups. In Proceedings of the 2001 IEEE Conference on Decision and Control, volume 3, 2968-2973. IEEE.

McMickell, M.B. and Goodwine, B. (2001). Reduction and controllability of symmetric distributed systems with robotic applications. In International Conference on Intelligent Robotics and Systems, volume 3, 1232-1236. IEEE/RSJ.

McMickell, M.B. and Goodwine, B. (2002). Reduction and controllability of robotic systems with drift. In
Proceedings of the 2002 IEEE International Conference on Robotics and Automation, volume 4, 3454-3460. IEEE.

McMickell, M.B. and Goodwine, B. (2003a). Reduced order motion planning for nonlinear symmetric distributed robotic systems. In Proceedings of the 2003 IEEE International Conference on Robotics and Automation, volume 3, 4228-4233. IEEE.

McMickell, M.B. and Goodwine, B. (2003b). Reduction and nonlinear controllability of symmetric distributed systems. International Journal of Control, 76(18), 18091822

McMickell, M.B. and Goodwine, B. (2007). Motion planning for nonlinear symmetric distributed robotic systems. International Journal of Robotics Research, 26(10), 1025-1041.

McMickell, M.B., Goodwine, B., and Montestruque, L.A. (2003). Micabot: A robotic platform for large-scale distributed robotics. In Proceedings of the 2003 IEEE International Conference on Robotics and Automation, volume 2, 1600-1605. IEEE.

Olfati-Saber, R. and Murray, R.M. (2002). Distributed cooperative control of multiple vehicle formations using structural potential functions. In IFAC World Congress, 346-352.

Olfati-Saber, R. and Murray, R.M. (2003). Consensus protocols for networks of dynamic agents. In Proceedings of the 2003 American Controls Conference.

Recht, B. and D'Andrea, R. (2004). Distributed control of systems over discrete groups. IEEE Transactions on Automatic Control, 49(9), 1446-1452.

Ren, W., Beard, R.W., and Atkins, E.M. (2005). A survey of consensus problems in multi-agent coordination. In Proceedings of the 2005 American Controls Conference, 1859-1864.

Ren, W., Beard, R.W., and Atkins, E.M. (2007). Information consensus in multivehicle cooperative control. IEEE Control Systems Magazine, 71-82.

Rimon, E. and Koditschek, D.E. (1992). Exact robot navigation using artificial potential functions. IEEE Transactions on Robotics and Automation, 8(5), 501518.

Rotman, J.J. (1995). An Introduction to the Theory of Groups. Springer-Verlag, fourth edition.

Sztipanovits, J., Koutsoukos, X., Karsai, G., Kottenstette, N., Antsaklis, P., Gupta, V., Goodwine, B., Baras, J., and Wang, S. (2012). Toward a science of cyber-physical system integration. Proceedings of the IEEE, 100(1), 2944.

Yoshizawa, T. (1966). Stability theory by Liapunov's second method. Mathematical Society of Japan (Tokyo). 